\title{
CURRENT DRIVE WITH FAST WAVES, ELECTRON CYCLOTRON WAVES, AND NEUTRAL INJECTION IN THE DIII-D TOKAMAK
}

R. PRATER, R.A. JAMES, ${ }^{*}$ C.C. PETTY, R.I. PINSKER,

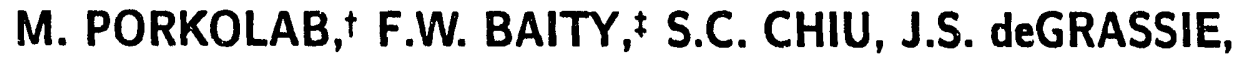
R.H. GOULDING, $\ddagger$ R.W. HARVEY, D.J. HOFFMANN, $\ddagger$ H. IKEZI, H. KAWASHIMA," Y.R. LIN-LIU, T.C. LUCE, and V. TRUKHIN ${ }^{b}$

This is a preprint of a paper to be presented at the European Topical Conference on RF Heating and Current Drive of Fusion Devices, July 7-10, 1992, Brussels, Belgium, and to be printed in the Proceedings.

Work supported by U.S. Department of Energy

Contract Nos. DE-AC03-89ER51114, DE-AC03-78ET51013, DE-AC02-76-CHO-3073, and DE-AC05-84-OR21400 * Lawrence Livermore National Laboratory, Livermore, California.

${ }^{\dagger}$ Massachusetts Institute of Technology, Cambridge, Massachusetts.

$\ddagger$ Oak Ridge National Laboratory, Oak Ridge, Tennessee.

"Japan Atomic Energy Research Institute, Japan.

'Kurchatov Institute, Russia.

GENERAL ATOMICS PROJECT 3466 SEPTEMBER 1992 


\title{
CURRENT DRIVE WITH FAST WAVES, ELECTRON CYCLOTRON WAVES, AND NEUTRAL INJECTION IN THE DI!I-D TOKAMAK
}

\author{
R. Prater, R.A. James," C.C. Petty, R.I. Pinsker, M. Porkolab, ${ }^{\dagger}$ \\ F.W. Baity, ${ }^{\ddagger}$ S.C. Chiv, J.S. DeGrassie, R.H. Goulding, ${ }^{\ddagger}$ R.W. Harvey, \\ D.J. Hoffman, H. Ikezi, H. Kawashima, Y.R. Lin-Liv, \\ T.C. LUCE, V. TRUKhIN' \\ General Atomics, San Diego, California, U.S.A. \\ "Lawrence Livermore National Laboratory. \\ tMassachusetts Institute of Technology. \\ Oak Ridge National Laboratory. \\ Japan Atomic Energy Research Institute. \\ 'Kurchatov Institute.
}

KEY WORDS: Plasma heating, current drive, fast wave current drive

\begin{abstract}
Current drive experiments have been performed on the DIII-D tokamak using fast waves, electron cyclotron waves, and neutral injection. Fast wave experiments were performed using a 4-strap antenna with $1 \mathrm{MW}$ of power at $60 \mathrm{MHz}$. These experiments showed effective heating of electrons, with a global heating efficiency equivalent to that of neutral injection even when the single pass damping was calculated to be as small as $5 \%$. The damping was probably due to the effect of multiple passes of the wave through the plasma. Fast wave current drive experiments were performed with a toroidally directional phasing of the antenna straps. Currents driven by fast wave current drive (FWCD) in the direction of the main plasma current of up to $100 \mathrm{kA}$ were found, not including a calculated $40 \mathrm{kA}$ of bootstrap current. Experiments with FWCD in the counter current direction shnwed little current drive. In both cases, changes in the sawtooth behavior and the internal inductance qualitatively support the measurement of FWCD. Experiments on electron cyclotron current drive have shown that $100 \mathrm{kA}$ of current can be driven by $1 \mathrm{MW}$ of power at $60 \mathrm{GHz}$. Calculations with a Fokker-Plands code show that electron cyclotron current drive (ECCD) can be well predicted when the effects of electron trapping and of the residual electric field are included. Experiments on driving current with neutral injection showed that effective current drive could be obtained and discharges with full current drive were demonstrated. Interestingly, all of these methods of current drive had about the same efficiency, $0.015 \times 10^{20} \mathrm{MA} / \mathrm{MW} / \mathrm{m}^{2}$.
\end{abstract}

\section{INTRODUCTION}

The ability to operate a reactor in steady state has long been viewed as a critical part of the feasibility of fusion. For a tokamak reactor, operation in steady state requires that a noninductive means of driving current be found. By using radio-frequency waves incident unidirectionally on the plasma, net electrical currents may be generated in the plasma (FisCH, 1987). Lower hybrid waves have been most successful in this rega d (UEHARA, 1989; SöLDNER, 1991), but electron cyclotron waves have also been shown to drive currents (START, 1982; ROBINSON, 1987; TANAKA, 1988; JAMES, 1990), although at lower efficiency. Fast waves hold the promise of current drive with an efficiency comparable to that of lower hybrid waves (CHIU, 1989), but without some of the drawbacks of lower hybrid techniques. (Efficiency is defined as the ratio of the driven current $I_{C D}$ in $\mathrm{MA}$ to the power required to drive that current $P_{C D}$ in $M W$, normalized by the plasma density $n_{e}$ in units of $10^{20} \mathrm{~m}^{-3}$ multiplied by the major radius of the tokamak $R$ in meters: $\eta=n_{e} I_{C D} R / P_{C D}$.) The physics of fast wave current drive (FWCD) in the ion cyclotron range of frequencies has not yet been fully demonstrated, and that is a topic of this paper. These results will be compared with those of electron cyclotron current drive (ECCD) (LUCE, 1991; JAMES, 1992) and neutral beam current drive (NBCD) (SIMONEN, 1988; NAVRATIL, 1991) on DIII-D.

Efficiency of current drive is a key issue, of course. If the efficiency is too low, then too large a fraction of the output power of a reactor must go into driving the plasma current. At present, it appears that the efficiency of current drive is marginal for driving all the plasma current in a tokamak reactor of standard design (for example, in the Conceptual Design Activity version of ITER (ITER, 1991)) without using a large fraction of the output power for the current drive. 
However, recent progress in the research program on tokamaks has identified several new motives for pursuing current drive. First, experiments on several large tokamaks have shown that the neoclassical bootstrap effect can drive a large fraction of the plasma current (ZARNSTORFF, 1988; MEADE, 1991; START, 1992; AKAOKA, 1991). (The bootstrap current is an electrical current in the plasma which flows in the toroidal direction as a consequence of radial density and temperature gradients.) Experiments on JT- 60 have shown that up to $80 \%$ of the plasma current can be obtained by heating alone (AKAOKA, 1991). This implies that a reduction may be made in the magnitude of the current which must be driven by external means. Second, regimes of improved confinement of energy are being developed on several tokamaks. When the H-mode was found, confinement doubled (WAGNER, 1982); with the VH-mode came another factor of two (JACKSON, 1991). Further improvements can be anticipated, based on theoretical constructs which have been developed to explain the improved modes of confinement (GROEBNER, 1991). Since energy confinement in these regimes is proportional to the plasma current, improved confinement implies that the plasma current may be reduced, for the same total sonfinement. As the plasma current is reduced, the efficiency of the power required to drive it becomes less important. And third, as current drive techniques are studied, it often becomes possible to find means of improving their efficiency.

Another important factor motivating research on current drive is the effect that the current density profile has on confinement and stability. Experiments on TFTR (MEADE, 1991) and DIII-D (TAYLOR, 1991) have suggested that the confinement may be improved through peaking of the current density profile, and further experiments on DIII-D have shown that the beta limit may be increased as well (STRAIT, 1991). While these experiments have relied on inductive techniques such as ramping the current or the elongation to modify the current profile, which are inherently transitory, it is expected that noninductive means such as if current drive, if applied in a manner to produce the same current profile, would be equally effective and applicable in steady state.

In this work, we report progress on driving plasma current with fast waves in the ion cyclotron range of frequencies $\left(2 \Omega_{H}<\omega<4 \Omega_{H}\right)$. Fast waves do not suffer the accessibility and strong damping limits that affect lower hybrid waves, which cannot penetrate to the conter of a large plasma with electron temperature above about $10 \mathrm{keV}$. Because the wavenumber parallel to the magnetic field $\left(k_{\|}\right)$of the fast wave is typically much smaller than that of the lower hybrid wave, the requirement of good coupling at the edge does not require that the antenna be so close to the plasma. However, the fast wave is weakly damped unless the electron beta is high (CHIU, 1989). This has impeded research on FWCD. The electron cyclotron heating (ECH) system on DIII-D offers a unique opportunity to study the physics of FWCD by providing independent control of the electron temperature.

The experiments presented here represent the first steps of the FWCD program. The experiments are meant to demonstrate the basic physics of the interaction of the fast wave with electrons and to advance the numerical models for fast wave heating and current drive by providing a body of experimental data for comparison. At the low if powers involved at this stage of the program, large noninductive currents are not expected. On DIII-D, we have the opportunity to compare the current drive using fast waves with that using electron cyclotron waves or neutral beams.

\section{EXPERIMENT}

The DIII-D tokamak is well suited to a study of FWCD. It has a flexible poloidal field coil system which facilitates operation in many configurations, including single- and double-null divertor and inside- and outside-wall limited. Double null divertor discharges with full size which fill the vacuun vessel and with small minor radius moved to the outboard side of the vacuum vessel have been used in these experiments. The major radius of full sized plasmas is $1.68 \mathrm{~m}$, the minor radius is $0.68 \mathrm{~m}$, and the elongation is between 1.4 for the small plasmas and 1.9 for the full sized plasmas. The plasma current in these experiments has been between 0.3 and 1.2 MA, and the toroidal field has been between 0.75 and $2.1 \mathrm{~T}$. The working gas is deuterium. The walls of the vacuum vessel are treated with the low- $Z$ materials boron or carbon to reduce the influx of metallic impurities. Boronization was particularly effective at reducing radiated power (to less than $30 \%$ of the input power) and at minimizing the density increase which accompanies fast wave heating.

The fast wave antenna occupies a pair of midplane ports on the outer wall of the vacuum vessel. It has four independertily phased current carrying straps spaced $22 \mathrm{~cm}$ between centers. The strajs are $11 \mathrm{~cm}$ wide and $45 \mathrm{~cm}$ high. The Faraday screen is an optically dense arrangement of two rows of liconel rods covered with a thin layer of TiC/TiN on their plasma-facing side. The front of the straps is $3.5 \mathrm{~cm}$ behind the adjacent graphite tiles on the vacuum vessel wall, and the front of the Faraday screen is $0.5 \mathrm{~cm}$ behind the tiles. Under normal divertor operation, the plasma separatrix lies 2 to $3 \mathrm{~cm}$ from the tile siurface. The antenna was made at Oak Ridge National Laboratory (GouldiNG, 1992; HOFFMAN, 1992).

In some of the results described in this report, the Faraday screen was removed. Faraday screens in general have a number of negative attributes. They are a source of impurities which sheath effects can inject into the plasma, they introduce some absorption and reflection of the wave polarization they are supposed to transmit, and unless the elements of the shields are oriented perfectly along the magnetic field lines they induce some of power in the unwanted polarization. The technical difficulties with a Faraday shield are even more severe: mechanically and thermally they constitute one of the more severe challenges to be found on the first wall of a tokamak. For reactors, these problems may not be soluble. Following the lead of the TEXTOR group, which showed that under special conditions an ICRF antenna could be operated without 
a Faraday shield (VAN Nieuwenhove, 1990), the FW'CD antenna in some of these experiments has been operated without the shield.

The antenna is driven by an if source with power up to $2 \mathrm{MW}$ and frequency of 30 to $60 \mathrm{MHz}$. Experiments on heating a hydrogen minority were performed at $32 \mathrm{MHz}$ at a field of $2 \mathrm{~T}$, and all FWCD experiments lised $60 \mathrm{MHz}$. The transmission line, tuning, and phasing scheme is described in detail in (MAYBERRY, 1992; Goulding, 1992; PINSKer, 1992). The first and third straps and the second and fourth straps are connected by coaxial lines of resonant electrical length which maintain the phase difference between those straps at 0 or $\pi$ radians. By means of two stub tuners and three phase shifters, it was possible to set the phase shift between adjacent antenna straps to $\pm \pi / 2$ radians, with approximately equal currents in each strap and with a good match between the $50 \Omega$ transmission line and the rf source.

At $60 \mathrm{MHz}$ and with the phase of the straps set to $(0, \pi, 0, \pi)$, the parallel index of refraction $n_{\|}$peaks at \pm 9 with subpeaks at \pm 2.5 , as shown in Fig. 1(a). The spectrum is symmetric around zero, so equal powers are launched in both toroidal directions. For $\pi / 2$ phase shift between straps, the $(0, \pi / 2, \pi, 3 \pi / 2)$ phasing, the spectrum shown in Fig. 1(b) is highly asymmetric toroidally, as needed for current drive. The peak of the forward spectrum is at $n_{\|}=5$. The spectra shown in Fig. 1 are for power coupled to the plasma for conditions typical of the DIII-D experiments through an evanescent layer which tends to selectively attenuate the high $n_{\|}$components. The resultant spectrum is sensitive to the details of the evanescent layer, particularly for the $\pi$ phasing. Geometric and other effects tend to shift $n_{\|}$to larger values near the plasma center.

The ECH is provided by the $60 \mathrm{GHz}$ system, which uses inside launch of the extraordinary mode. In most of the experiments reported here, the ECH power incident on the plasma is in the range of 0.6 to $1.2 \mathrm{MW}$. The inside launch antennas have a Gaussian radiation pattern of characteristic angle $10^{\circ}$, and they are located on the in-board vessel wall $13 \mathrm{~cm}$ above the midplane of the vessel. The antennas radiate with the center of the radiation pattern at an angle of $+15^{\circ}$ from the radial, so when the ECH is being used to heat the plasma for FWCD experiments, the ECH power drives some plasma current which must be accounted for in the analysis. Ray tracing calculations using many rays to simulate the radiation pattern show that the first pass heating efficiency drops rapidly above a critical density for characteristic density profiles, due to the combined effects of refraction and reduced opacity. For fundamental heating at $2.14 \mathrm{~T}$, the characteristic line-averaged density is about $5.5 \times 10^{19} \mathrm{~m}^{-3}$, while for second harmonic heating at $1.07 \mathrm{~T}$ the characteristic density is about $1.4 \times 10^{19} \mathrm{~m}^{-3}$.

High power neutral injection is provided by four beamlines, each with two neutral beam sources. Operated in deuterium at a voltage of 77 to $80 \mathrm{kV}$, each source can deliver up to $2.5 \mathrm{MW}$ of power, for a total heating power of $20 \mathrm{MW}$. In deuterium, $78 \%$ of the power is in the full energy component, $16 \%$ in the half energy component, and $6 \%$ in the third energy component. The more tangential beams intersect the axis of the plasma at an angle of $47^{\circ}$, and the less tangential beams at an angle of $63^{\circ}$. Maximum pulse length for these sources is 5 sec.

\section{FAST WAVE HEATING}

Fast (magnetosonic) waves are Alfvén waves which in a tokamak propagate primarily radially. Fast waves can be absorbed near the ion cyclotron harmonic resonances, or directly by electrons which satisfy the resonance condition

$$
\boldsymbol{\omega}-\boldsymbol{k}_{\|} \boldsymbol{v}_{\| \boldsymbol{e}}=0
$$
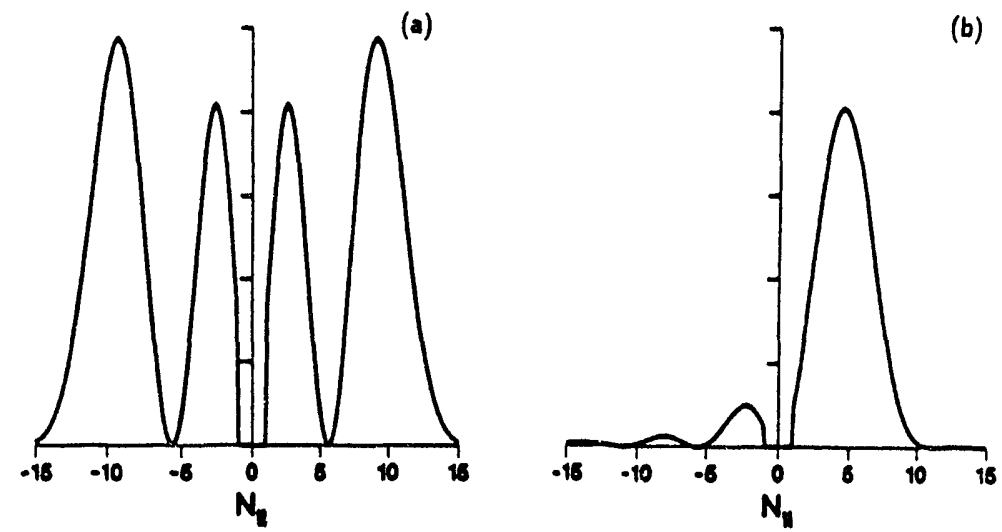

Fig. 1. Spectrum of power coupled into the plasma by the FW antenna for phasing (a) $(0, \pi, 0, \pi)$ and $(b)(0, \pi / 2, \pi, 3 \pi / 2)$, calculated for density profiles typical of Dill-D. 
(Here, $\omega$ is the applied frequency, $k_{\|}$is the wavenumber parallel to the magnetic field $B$, and $v_{\| e}$ is the component of the electron velocity parallel to $B$.) For a Maxwellian distribution, the imaginary part of the wavenumber is given by (CHIU, 1989; PORKOLAB, 1992)

$$
k_{\perp I m}=k_{\perp R e}\left(\pi^{1 / 2} / 4\right) \beta_{e} \xi_{e} \exp \left(-\xi_{e}^{2}\right)\left(1+\alpha^{-2}\right)
$$

where $k_{\perp R e} \approx \omega / v_{A}, v_{A}$ is the Alfvén speed, $\beta_{e}=2 \mu_{0} n_{e} k T_{e} / B^{2}$ is the electron betia, $\xi_{e}=\omega / k_{\|} v_{t e}$ is the ratio of the parallel phase velocity of the wave to the thermal velocity of the electrons, and $\alpha$ is a function which represents the cross coupling between transit time magnetic pumping (TTMP) and Landau damping in a plasma with finite temperature and frequency (PORKOLAB, 1992). For the experimental conditions of the experiments presented here, the magnitude of $\alpha^{-2}$ is less than unity.

The damping described by Eq. (1) is maximum when $\xi_{e} \approx 1 / \sqrt{2}$, where $\xi_{e}$ can be written $\xi_{e}=c / n_{\|} v_{t e}$ and $n_{\|}$is the parallel index of refraction. In practice the $n_{\|}$is upshifted from that launched by the antenna through the effects of toroidal geometry, for which fixed $k_{\|} R$ causes an increase in $n_{\|}$of about $40 \%$ as the wave travels from the antenna to the plasma center, and a smaller upshift due to the poloidal magnetic field. Including the effect of the geometric shift, optimum damping corresponds to a parallel index of refraction at the antenna $n_{\| 0}$ which satisfies

$$
n_{\| 0} \approx \sqrt{\frac{250}{T_{e}(\mathrm{keV})}} .
$$

For the experimental conditions present in DIII-D for these experiments, the magnitude of $k_{\perp I m}$ given by Eq. (2) is rather small. Attenuation of a ray passing through the plasma is given by

$$
A=1-\exp \left(-2 \int_{-a}^{a} k_{\perp I m} d r\right)
$$

where $A$ is the ratio of the absorbed power to the incident power of the wave for a single pass of the wave through the plasma and $r$ is the distance along the projection of a ray path onto a surface of constant azimuth. For the experiments presented here, $A$ is less than $30 \%$, so the ray extinction is approximately proportional to $k_{\perp I m}$. From Eq. (2), the absorption is therefore proportional to $B^{-3}$. This strong dependence on the magnetic field motivates experiments at low field. For the frequency and antenna geometry used in the experiments, the absorption also increases strongly with electron temperature.

Experiments on DIII-D regarding direct heating of electrons through Landau damping and transit time magnetic pumping have shown highly efficient heating (PRATER, 1992; PETTY, 1992a,b,c), even though the damping calculated from Eq. (3) for a single pass of the ray through the plasma is small. The experiments were performed using $(0, \pi, 0, \pi)$ phasing of the antenna. The heating efficiency for fast waves was determined by comparing the increase in stored energy or the initial rate of rise of the stored energy for fast wave heating with that for electron cyclotron heating or neutral injection, for both of which the heating is well characterized. The comparison was done at different times in the same discharge, at nearly the same level of input power. An example discharge in which FW heating is compared with ECH is shown in Fig. 2, which will be discussed in more detail below. The comparison showed that, within an uncertainty of about $\pm 15 \%, 95 \%$ of the fast wave power incident on the plasma was absorbed. A corroborating observation is that a transition to the H-mode regime of improved confinement was reliably obtained with $750 \mathrm{~kW}$ of fast wave power at a toroidal field of $1.0 \mathrm{~T}$ and density of $1.7 \times 10^{10} \mathrm{~m}^{-3}$ (PRATER, 1992; PETTY, 1992a,b,c). This power is at or below the minimum level of power needed to obtain a transition to the H-mode in DIII-D using neutral beam injection (CARLSTROM, 1989) or electron cyclotron heating (LOHR, 1988), for similar plasma conditions.

A critical issue is whether the damping of the wave is due to absorption by electrons directly or by ions, since damping by ions will probably not contribute to current drive. Absorption by ions is expected to be significant only near the iow harm.unics of the cyclotron resonance. At $60 \mathrm{MHz}$, the second harmonic of hydrogen lies near the center of the plasina for a toroidal field of $2 \mathrm{~T}$, so some absorption by hydrogen ions is possible. The fraction of hydrogen ion: in these experiments was about $2 \%$ (as measured at the plasma edge), at which concentration an ionic contribution to the damping of order $20 \%$ of the total wave damping is expected from calculations with a full wave code (BoNol1, 1992). Damping by ions is calculated to be much smaller at other toroidal fields. Damping by ions is therefore not expected to have a major effect on the power balance in these experiments.

The time behavior of the electron temperature supports the theoretical expectation that absorption by ions is weak. For experiments at a toroidal field of $2 \mathrm{~T}$, the condition most likely to produce ion heating since the second harmonic of the hydrogen resonance is central, the electron temperature rose much more rapidly than the ion temperature, as shown in Fig. 2 of (PRATER, 1992). In contrast, if the absorption were due to 

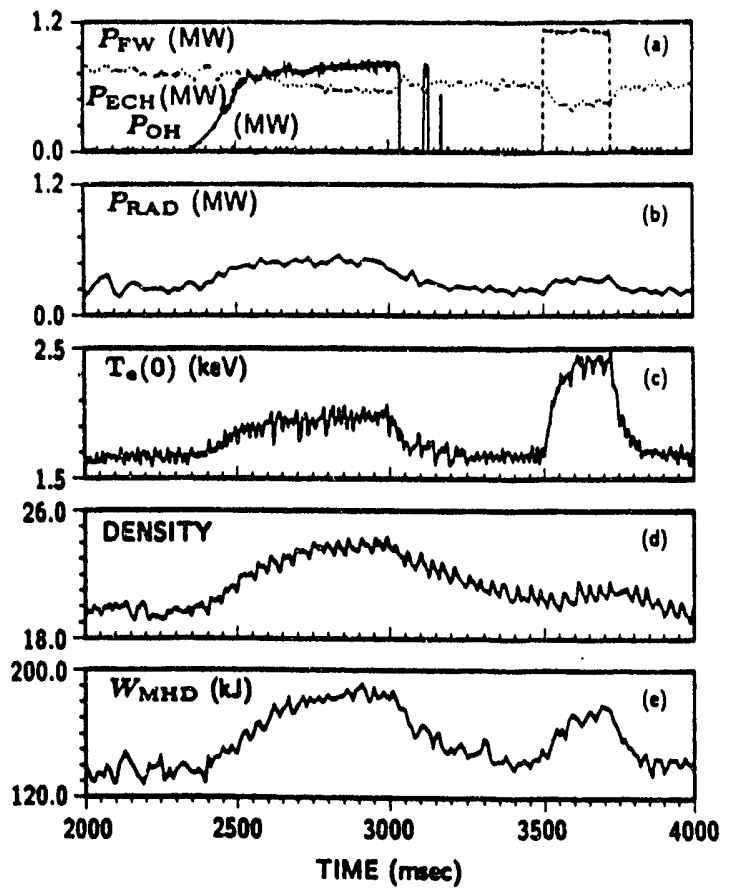

Fig. 2. Behovior of a discharge with fast wave heating using $(0, \pi, 0, \pi)$ phasing of the antenna. For this discharge, the antenna has no Faraday shield. Shown are (a) fast wave power, ECH power (dashed line), and Ohmic power (dotted line); (b) total radiated power; (c) central electron temperature; (d) line-sveraged density in units of $10^{12} \mathrm{~cm}^{-3}$; and (e) plasma kinetic energy. The toroidal field is $1.0 \mathrm{~T}$ and the plasma current is $400 \mathrm{kA}$.

second harmonic heating of the hydrogen minority, an energetic tail of the hydrogen ion distribution should be generated which would slow down on and heat both the bulk ions and the electrons. The presence of the tail would also be apparent as a difference between the stored energy determined from MHD analysis and that from diamagnetic effects. But the experiments showed very little systematic variation between the MHD and the diamagnetic energy. Additionally, measurements of energetic neutrals from charge exchange and direct measurements of the location of the electron heating performed by modulating the FW power (PETTY, 1992A; PRATER, 1992) support the view that absorption by ions is playing only a small role in the power balance for these experimental conditions. On the basis of transport modeling, the increase in ion temperature can be attributed to thermal conduction from the electrons.

The plasma heating produced by the fast waves does not exhibit the strong inverse dependence on toroidal field of the single pass damping which follows from Eqs. (2) and (4). This was found by measuring the confinement time for the range 0.8 to $2 \mathrm{~T}$ in toroidal field, as shown in Fig. 3. In order to exhibit more clearly the dependence of the heating efficiency on toroidal field, as opposed to the dependence of the background confinement, the measured confinement times of Fig. 3 were divided by the confinement times given by the ITER-89P L-mode scaling (ITER TEAM, 1989). The confinement in DIII-D in L-mode using NBI, ECH (LUCE, 1991), or ICH (PETTY, 199D) agrees well with the ITER-89P scaling, so this normalization procedure is a valid means of removing the background variation of confinement with toroidal field. Figure 3 shows that the normalized confinement, which can be interpreted as the effective heating rate to the extent that the energy confinement scales with the weak ITER-89P toroidal field scaling, is uniformly high over the full range of toroidal field.

The data in Fig. 3 were taken for plasmas with reduced minor radius of $0.48 \mathrm{~m}$ and with increased major radius so that the outboard edge of the plasma was close to the antenna. For this configuration, at a field of $1.71 \mathrm{~T}$, the plasma lies between the second harmonic hydrogen rescnance on the inboard side and the third harmonic hydrogen resonance on the outboard side, with no low order cyclotron resonances within the bulk of the plasma. Figure 3 shows no reduction in heating efficiency for this case compared to the $2 \mathrm{~T}$ case where the second harmonic hydrogen resonance is central.

The data in Figs. 2 and 3 show that a large fraction of the power is absorbed. As theory predicts a very low damping decrement in a single pass (the filled circles in Fig. 3), one must conclude that the damping is taking place through multiple passes of the rays through the plasma or that the theory underestimates the damping. To test the multiple pass idea, the characteristics of the propagation of the wave were studied using probes which measure the poloidal and the toroidal components of the rf magnetic fields. The probes were located $\pm 135^{\circ}$ toroidally away from the antenna and $20 \mathrm{~cm}$ outside the plasma boundary. Large phase shifts of $(10-25) \times 2 \pi$ radians between the launched wave and the received wave were found. The phase shifts were dependent on the density, roughly proportional to $\sqrt{n_{e}}$ which suggests propagation delay's determined by the Alfvén speed rather than an eigenmode with fixed toroidal mode number of 


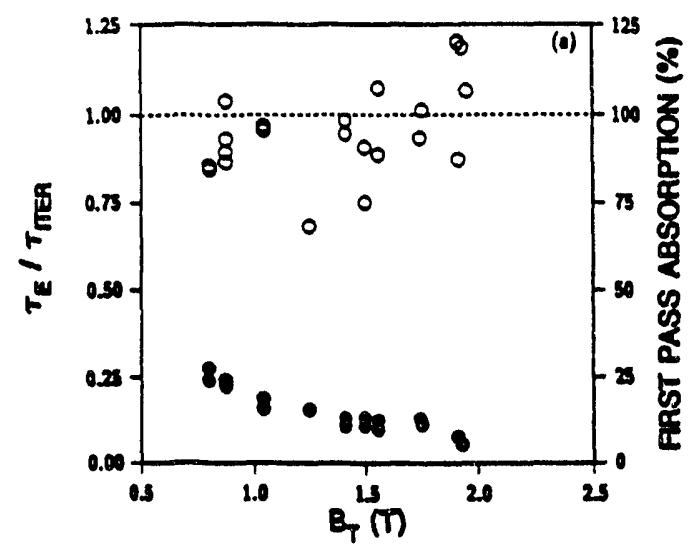

Fig. 3. The observed energy confinement time normalized by the ITER89-P L-mode confinement scaling as a function of toroidal field (empty circles). Also shown is the calculated single-pass absorption for the conditions of the experiments. The plasma is in a double null divertor configuration with reduced minor radius $(0.48 \mathrm{~m})$, the elongation is 1.4 , the plasma current is $0.3 \mathrm{MA}$, and the density is $2-2.5 \times 10^{10} \mathrm{~m}^{-3}$.

about 35 as imposed by the antenna. The amplitudes of the detecied signals changed less than $20 \%$ for changes in density of order $50 \%$, with no fine structure that could be attributed to eigenmodes except at the very lowest densities attainable. On the basis of these measurements, our working hypothesis is that the damping takes place through multiple passes of the rays, and eigenmodes do not play a large role.

In order to test the heating effectiveness and other aspects of an antenna without a Faraday shield, some experiments were done with the Faraday shield removed, following the pioneering work of (VAN NIEWENHOVE, 1990) on the TEXTOR tokamak. The antenna was not designed for operation without a shield, so the configuration was not ideal. In particular, part of the stripline that feeds the antenna was exposed to the plasma. This may result in electric fields in the plasma which set up convective cells and enhance losses in the scrape-off layer. Additionally, plasma may enter the strip-line and add dissipation or change th'. impedance.

In the experiment, a strong dependence of antenna loading on power was otserved: at low power, anomalously large loading was measured, which decreased towards the levels seen previously with the Faraday screen as the power was raised. The loading appeared to return to normal levels at a net power of about $100 \mathrm{~kW}$. This phenomenon may result from ponderomotive forces; the very luw density plasma which might enter the antenna box can support electrostatic modes (electron plasma waves) which may cause the large loading at low power. As the power is raised, the tenuous plasma is pushed out of the box by the ponderomotive force.

The power handling of the antenna without a Faraday screen appeared to be significantly inferior to the screened antenna. This property of screenless operation has also been seen on TEXTOR (VAN NIEWENHOVE, 1992), where it was alleviated by an extensive program of antenna conditioning in vacuum. Unfortunately, time limitations prevented such conditioning procedures from being carried out. At power levels in excess of about $0.6 \mathrm{MW}$, pulses usually ended in a series of antenna arcs, visible on a video view of the antenna surface, and resulting in a sudden influx of copper and other metal impurities into the discharge. However, heating with $(0, \pi, 0, \pi)$ antenna phasing was observed to be approximately as efficient as had been obtained with the screen in place, as long as the pulse was free of antenna arcing; furthermore, no significant If-specific impurity influx was observed except during antenna arcing, and radiated power is low. This behavior is shown in Fig. 3 , in which FW heating is compared with ECH at a comparable power level.

\section{FAST WAVE CURRENT DRIVE}

Fast wave current drive experimernts were performed by phasing the straps of the antenna $(0, \pi / 2, \pi, 3 \pi / 2)$ to launch a wave which travels primarily in one toroidal direction. For this phasing the traveling wave spectrum is shown in Fig. 1(b). The peak in $n_{\|}$coupled at the edge of the plasma is 5 to 5.5 , which makes the temperature for optimum coupling 8 to $10 \mathrm{keV}$, from Eq. 3; however, significant damping can take place even for $\xi_{e}$ twice the optimum value, which corresponds to a temperature of 2 to $3 \mathrm{keV}$. Power up to $1.1 \mathrm{MW}$ has been coupled with this phasing. The tuning and phasing system described (Mayberry, 1992; Goulding, 1992; PINSKer, 1992) was able to maintain the desired phasing over a wide range of plasma conditions. All current drive experiments were done with the Faraday screen in place.

\section{Analysis Technique}

The application of fast wave power is accompanied by a drop in the loop voltage due to an increase in plasma conductivity caused by increased electron temperature, as well as to current drive. In experiments 
to date, the full plasma current has not been driven by if. The fraction of the current due to current drive is found by comparing the loop voltage on the plasma necessary to maintain constant plasma current with that calculated from measurements of profiles of plasma temperature and $Z_{\text {eff }}$, assuming neoclassical resistivity and including the effect of the bootstrap current. The current driven by $\mathrm{rf}$ is then given by (JAMES, 1992)

$$
I_{r f}=\frac{V_{l}^{*}-V_{0}}{V_{l}^{*}}\left(I_{p}-I_{b}\right)
$$

where $V_{k}$ is the loop voltage at the surface of the plasma determined experimentally, $V_{l}^{*}$ is the loop voltage calculated from neoclassical resistivity without current drive, $I_{p}$ is the plasma current, and $I_{b}$ is the calculated bootstrap current. Equation (5) has been benchmarked against quasi-steady state discharges in DIII-D using ohmic heating, ECH, or neutral injection, and the typical difference between the measured loop voltage and the loop voltage calculated from Eq. (5) is $\pm 0.03 \mathrm{~V}$ after subtracting the calculated ECCD or NBCD. This is the same technique which was used previously for measurements of ECCD on DIII-D (JAMES, 1992).

Equation (5) zay be applied only when conditions are in a steady state. Following Bonoli (BONOLI, 1988), the surface voltage is related to the magnetic fluxes by

$$
V_{1}=-\frac{\partial \Phi_{O H}}{\partial t}-\frac{\partial \Phi_{p}}{\partial t}-\frac{\partial \Phi_{E F}}{\partial t}-\frac{\partial \Phi_{V S}}{\partial t}
$$

where $\Phi_{O B}$ is the flux due to the current in the ohmic heating coil, $\Phi_{p}\left(I_{p}\right)$ is the magnetic flux in the plasma, $\Phi_{E F}$ is the flux at the plasma surface due to currents in the poloidal field coils, and $\Phi_{V s}$ is the flux due to eddy currents in the vacuum vessel walls.

The condition under which Eq. (5) is applicable is that the term $-\partial \Phi_{O B} / \partial t$ dominate the right hand side of Eq. (6). The second term on the rhs of Eq. (6) can be made small by picking times for the analysis when the time derivative of $I_{p}$ is small. The last term in Eq. (6) is always small in these experiments. The term due to the poloidal field coils can be written for a circular plasma of large aspect ratio as (BONOLI, 1988)

$$
\frac{\partial \Phi_{E F}}{\partial t}=10^{4} M_{\ell, E F} \frac{d}{d t}\left\{\frac{-\mu_{0} I_{p}}{4 \pi R_{0}}\left[\ln \left(8 R_{0} / a\right)+\beta_{p}(a)+\ell_{i} / 2-3 / 2\right]\right\}
$$

where $M_{\ell, E F}$ is the murual inductance between the plasma and the poloidal field coils. Since the plasma in DIII-D is not circular in cross-section or large in aspect ratio, Eq. (7) does not strictly apply; however, Eq. (7) may be used as an approximation to select the best times (i.e., when $\partial \Phi_{E F} / \partial t \approx 0$ ) during a discharge for analysis.

The measured loop voltage must be corrected for time changing flux between the measuring lorp and the plasma. The surface voltage $V_{0}$ is related to the measured loop voltage $V_{l}$ by

$$
V_{l}=V_{l}-\frac{\partial \Delta \Phi_{\bullet}}{\partial t}
$$

Here, $\Delta \Phi$, is the flux between the surface of the plasma and the location where the loop voltage is measured. The flux $\Delta \Phi$, is determined from magnetic measurements by the EFIT (LAO, 1985) code.

\section{Experiment: Co-FWCD}

Results from a discharge with the antenna phased to drive current in the same direction as the Ohmic current are shown in Fig. 4. The $60 \mathrm{MHz}$ fast wave power is $1 \mathrm{MW}$ with $\pi / 2$ phasing (co-FWCD). Preheating with ECH at $0.5 \mathrm{MW}$ overlaps the first $400 \mathrm{msec}$ of the fast wave pulse. Neutral beams are injected for $20 \mathrm{msec}$ pulses in order to gather data on the ion temperature from the charge exchange recombination diagnostic. The plasma current was kept fixed at $395 \mathrm{kA}$. A substantial increase in $T_{e}$ and drop in loop voltage takes place due to electron heating when the ECH is initiated, and a further drop takes place when the FWCD . power is added. Figure $4(\mathrm{~d})$ shows the quantity $\left[\ln (8 R / a)+\beta_{p}+\ell_{i} / 2-3 / 2\right]$ from Eq. (7). The analysis is performed at two time slices for which Fig. 4(d) shows that $\partial \Phi_{E F} / \partial t \approx 0:$ at $2450 \mathrm{msec}$, during FWCD plus ECH, and at $2900 \mathrm{msec}$, during FWCD only.

Analysis of the loop voltage shows that the measured surface loop voltage of Fig. 4(e) lies well below the voltage $V_{\text {: }}^{*}$ which is calculated from neoclassical resistivity for the measured profiles using the ONETWO 

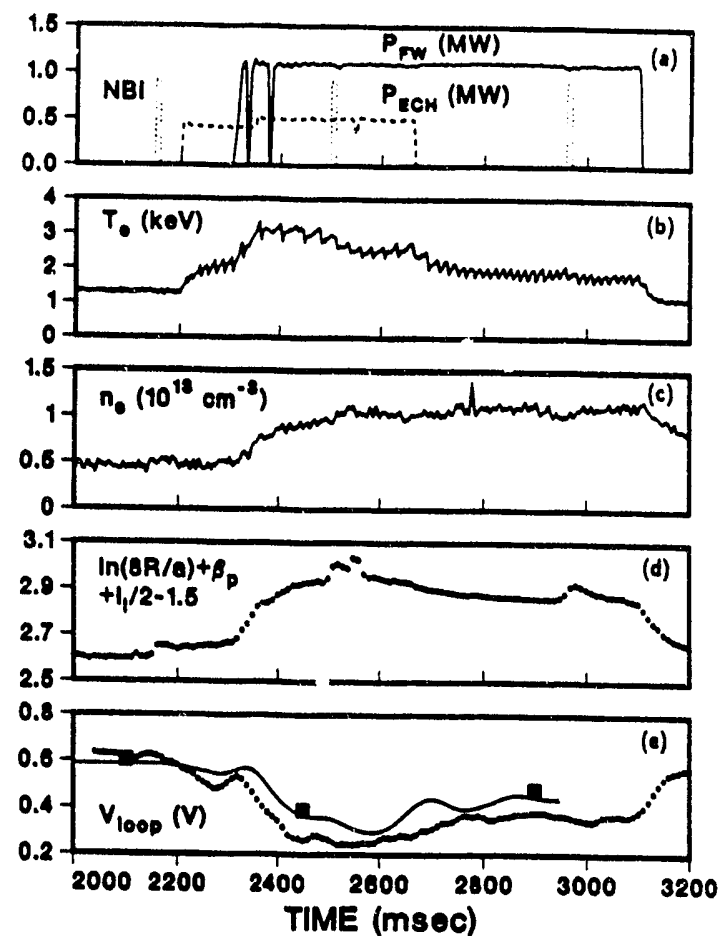

Fig. 4. Behavior of a discharge with co-current drive phasing. The toroidal field is $1.1 \mathrm{~T}$ and the plasma current is $395 \mathrm{kA}$. (a) Coupled FW and ECH power, and timing of injection of a $2 \mathrm{MW}$ neutral beam; (b) electron tempersture by electron cyclotron emission from a normalized radius of 0.1 ; (c) line-averaged density; (d) the calculated quantity $\ln (8 R / a)+$ $\beta_{p}+\ell_{i} / 2-1.5$; and (e) measured loop voltage corrected according to Eq. (8) (filled circles) and the loop voltage calculated from the profiles of temperature, $Z_{\text {eff, and density (filled }}$ squares). Also shown is the loop voltage calculated by a time-dependent simulation of the discharge (eolid line).

transport code [the filled squares in Fig. 4(e)]. This discrepancy indicates the presence of $117 \pm 20 \mathrm{kA}$ of If current drive, plus a calculated bootstrap c-rent of $44 \mathrm{kA}$, during FWCD plus ECH, and $81 \pm 20 \mathrm{kA}$ of driven current plus $41 \mathrm{kA}$ of calculated bootstrap current during FWCD alone. (Very similar results were obtained by analyzing these same data, using analytic expressions to model the profiles and equilibrium, by D. Ehst using the RIP code at Argonne National Laboratory.) The contribution to the rf driven current from ECCD was estimated at $23 \mathrm{kA}$ from calculations of current drive with the ray tracing code TORAY, including corrections estimated with Fokker-Planck calculations for the remaining Ohmic electric field. These results are summarized in Table I.

Also shown in Fig. 4(e) is the surface voltage developed by a time-dependent simulation of the discharge. In this simulation, the profiles of $T_{e}$ and $n_{e}$, which are measured every $50 \mathrm{msec}$ by Thomson scattering, are input, and for times between the Thomson times the profiles are determined by interpolation. The reasonable assumption is made that the toroidal electric field is fully penetrated during the Ohmic phase before the if power, and no if current drive is included in the simulation (but bootstrap current is included). The ONETWO transport code is used to evolve the electric field at the surface of the plasma consistent with the time history of $n_{e}, T_{e}$, and $Z_{e f f}$, using Maxwell's equations for a conducting medium. Difference between the simulated loop voltage and the loop voltage calculated for a particular set of profiles at a particular time is due to transient effects related to the relaxation of the radial profile of electric field. The agreement in loop voltage between the simulation (solid line) and the static calculations (filled squares) in Fig. 4(e) indicates that the loop voltage is fully equilibrated across the plasma for the times of the analysis.

The magnitude of the current driven by of is proportional to the rf power and to the inverse of the density (i.e., to the powes per particle), as shown in Fig. 5 . The slope of a line from the origin through a point is the normalized efficiency $\eta$ of the current drive divided by the major radius $R$. The data points with combined FWCD and ECH lie above those with FWCD only (i.e., at higher efficiency), probably due to $T_{e}$, as well as the additional ECCD.

The magnitude of the measured FWCD component is in relatively good agreement with theory, as shown in Fig. 6. In this figure, the theoretical $I_{F W}$ is determined from the CURRAY code. This code follows rays across the plasma integrating the absorption using the expression derived in (CHIU, 1992). Rays are followed for several traverses of the plasma, assuming full specular reflection at the plasma boundary. Typically, 35 rays are used to simulate the $k_{\|}$spectrum and the vertical extent of the antenna. All of the experimental data points are for cases without ECH, except for the point for which. $I_{F W}$ from theory is $120 \mathrm{kA}$. (This point may be low due to an overestimate of the ECCD.) Within errors, the experimentally 
TABLE I

Parameters Determined for Two Discharges, One With Co-FWCD

(the discharge of Fig. 4) and One With Counter-FWCD (the discharge of Fig. 9).

For Each Discharge, Time Slices With and Without ECCD are Shown.

\begin{tabular}{lllll}
\hline & \multicolumn{2}{c}{ Co-FWCD } & \multicolumn{2}{c}{ Counter-FWCD } \\
\cline { 2 - 5 } & FW + ECH & FW & FW + ECH & FW \\
\hline Total current, kA & 395 & 393 & 397 & 393 \\
Ohmic current, kA & 234 & 271 & 319 & 354 \\
Bootstrap current, kA & 44 & 41 & 43 & 39 \\
RF current drive, kA & $117 \pm 20$ & 81 & $35 \pm 35$ & $0 \pm 25$ \\
EC,CD, kA & 23 & 0 & 17 & 0 \\
FV.ICD, kA & 94 & 81 & $18 \pm 35$ & $0 \pm 25$ \\
Ray tracing FWCD & 113 & 65 & -54 & -38 \\
\hline $\bar{n}_{e}\left(10^{10} \mathrm{~m}^{-3}\right)$ & 0.94 & 1.08 & 1.17 & 1.24 \\
$T_{e}(k e V)$ & 3.63 & 2.05 & 2.41 & 2.08 \\
$Z_{\text {eft }}$ & 4.62 & 3.57 & 3.49 & 3.15 \\
Loop voltage & 0.26 & 0.37 & 0.26 & 0.46 \\
\hline
\end{tabular}

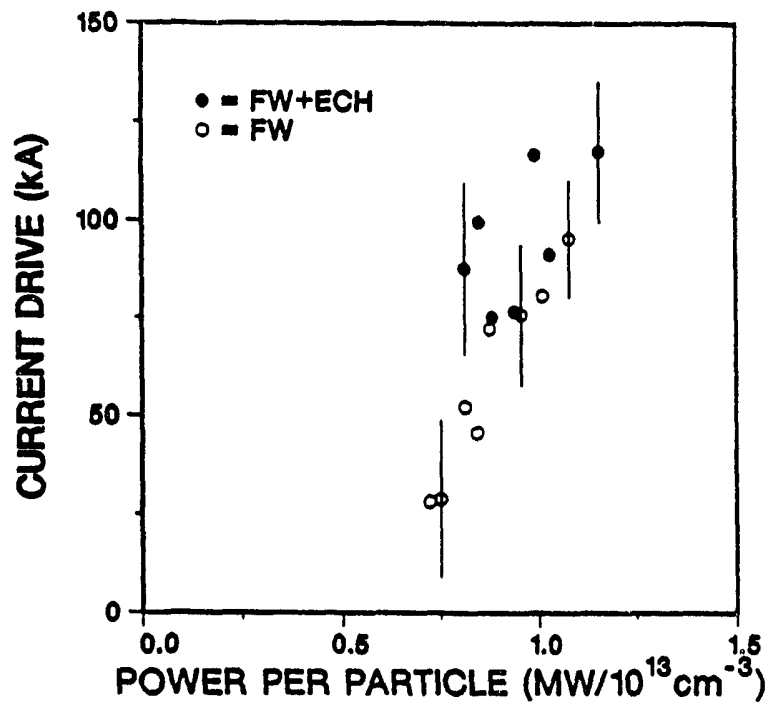

Fig. 5. RF current drive as a function of $f$ power per particle. The filled squares are for FWCD only and the empty squares are for FWCD plus ECCD. The toroidal field was $1.1 \mathrm{~T}$ and the plasma current was $400 \mathrm{kA}$.

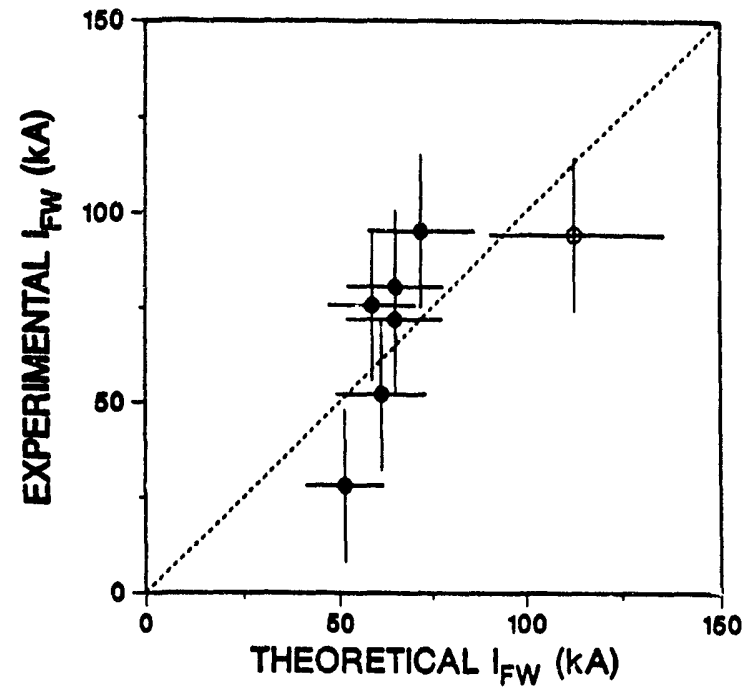

Fig. 6. Fast wave current drive determined experimentally vs the calculated current drive. The filled circles are without ECCD, and the empty circle has $500 \mathrm{~kW}$ of ECCD. The toroidal field is $1.1 \mathrm{~T}$ and the total plasma current is $400 \mathrm{kA}$. 
determined FWCD is consistent with the theoretical FWCD. Higher power FWCD experiments are needed to confirm any trends in the data.

In addition to measurement of the total current driven by the fast waves, the experiment provides information about the change in the current density profile. The radius of the sawtooth inversion layer is found to increase as the measured of current drive increases, as shown in Fig. 7, implying that due to the If current drive the current density within the $q=1$ surface is larger than that for pure Ohmic heating. Also, the internal inductance $\ell_{i}$ determined by the equilibrium code EFIT increases with fast wave power, from about 1.6 during Ohmic heating to 1.65 for $0.8 \mathrm{MW}$ of FW power and 1.7 for $1.0 \mathrm{MW}$, also implying that the current density profile is more peaked. While these changes in $l_{i}$ are small, they are repeatable and systematic. Changes of this nature are not found using ECH alone or FW heating with symmetric phasing of the antenna. Changes in $l_{i}$ or in the sawtooth inversion radius are negligible when the antenna phasing is symmetric and current drive is not expected.

These observations are qualitatively consistent with the view that the FWCD is responsible for increasing the peaking of the current density profile. The current density due to FW CD which is calculated by the CURRAY code for the discharge of Fig. 4 is shown in Fig. 8. The profile of FWCD is highly peaked, and nearly all of the driven current lies within the $q=1$ surface. For comparison, the equilibrium current density which is found by the EFIT code for the conditions of the experiment is about $120 \mathrm{~A} / \mathrm{cm}^{2}$, much smaller than the peak of the calculated profile for FWCD. While the magnitude of the peak in the calculated profile is not too important, since it represents only a tiny area and since the calculation does not include the effect of sawteeth which will certainly broaden any narrow peaks, the calculated average density of FWCD within the $q=1$ surface is about $70 \mathrm{~A} / \mathrm{cm}^{2}$. This current density is comparable to the equilibrium current density, so significant effects on the sawteeth should be expected. Similarly, the current profile of Fig. 8 is much narrower than the equilibrium profile of current density, so an increase in internal inductance with the application of FWCD is expected.

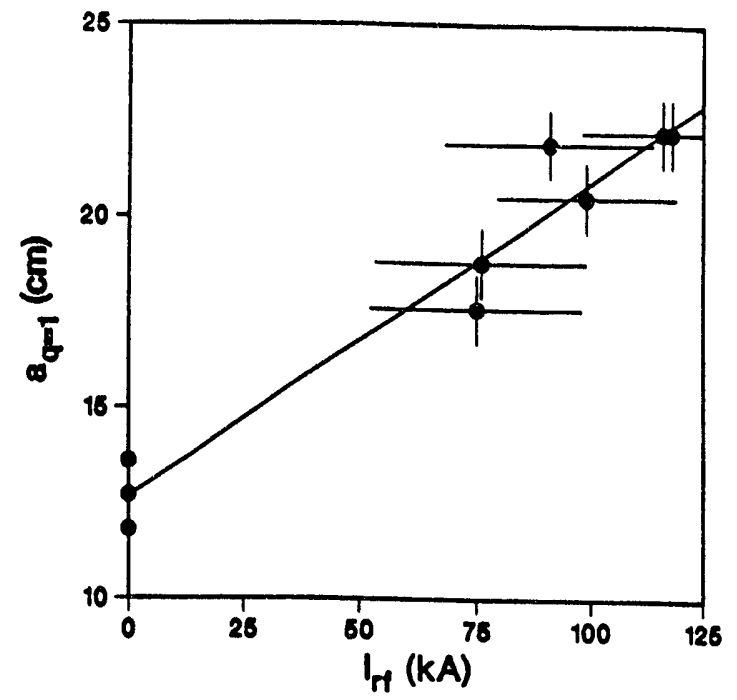

Fig. 7. Minor radius of the sawtooth inversion layer as a function of the measured if curreni drive (FWCD plus ECCD). The toroidal field is $1.1 \mathrm{~T}$ and the total plasma current is $400 \mathrm{kA}$.

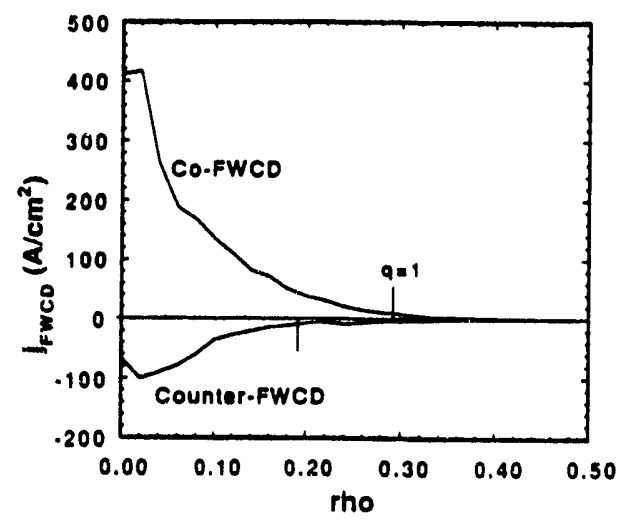

Fig. 8. Current density as a function of normalized poloidal magnetic flux, calculated using the CURRAY code for the Cs,-FWCD discharge of Fig. 4 and for the counter-FWCD discharge of Fig. 9. The locations of the $q=1$ surfaces for these two discharges are indicated. 
Counter current experiments are done by phasing the antenna straps at $(0,-\pi / 2,-\pi,-3 \pi / 2)$ so that the fast wave travels in the direction which would drive plasma current in the direction opposite that induced by the Ohmic transformer. In these experiments the ECCD remains in the co-current direction.

Clear differences are observed between counter-FWCD and co-FWCD. Figure 9 shows data from a discharge with counter-FWCD and ECH preheating. When the FW power starts, the electron temperature rises rapidly to $4 \mathrm{keV}$, as in Fig. 9(b). During the rise the sawteeth are very small or fully suppressed, until a large sawtooth crash occurs and the peak temperature begins falling. As the temperature falls, normal sawteeth set in and clamp the temperature at $2 \mathrm{keV}$. This behavior of the electron temperature and the sawteeth is characteristic of counter-FWCD discharges, and it does not occur for co-FWCD (as in Fig. 4).

The loop voltage during counter-FWCD, the circles in Fig. 9(d), shows a drop when the FW power is applied. In previous calculations of the current drive (PRATER, 1992) the incorrect assumption was made that the loop voltage at the edge of the plasma was nearly equal to the loop voltage at the plasma center. Under this assumption, the measured loop voltage was considerably below the voltage needed to sustain the plasma current under neoclassical resistivity, shown as the square points in Fig. 9(d). This was attributed to a forward current drive, instead of the expected counter current drive.

However, a time-dependent simulation of this discharge using the ONETWO code was performed which showed that the loop voltage is not well equilibrated across the plasma cross-section for the counter-FWCD cases. The simulation is similar to that performed for the co-FWCD case discussed above, which showed that in the co-FWCD case the simulated loop voltage agreed with that calculated from static profiles of temperature, density, and $Z_{\mathrm{e} f f}$. In the counter-FWCD case, the simulated loop voltage [tne solid line in Fig. 9(d)] does not agree well with the static equilibrium calculation [filled square in Fig. 9(d)] at $2500 \mathrm{msec}$ In this discharge, the large excursion in central electron temperature caused by the temporary suppression of the sawteeth apparently has a strong effect or the behavior of the surface voltage 100 to $150 \mathrm{msec}$ later.

By this analysis, the current driven by $\mathrm{ff}$ is small, since the measured loop voltage agrees well with the loop voltage at the plasma surface determined by the time-dependent simulation, as shown in Fig. 9(d).
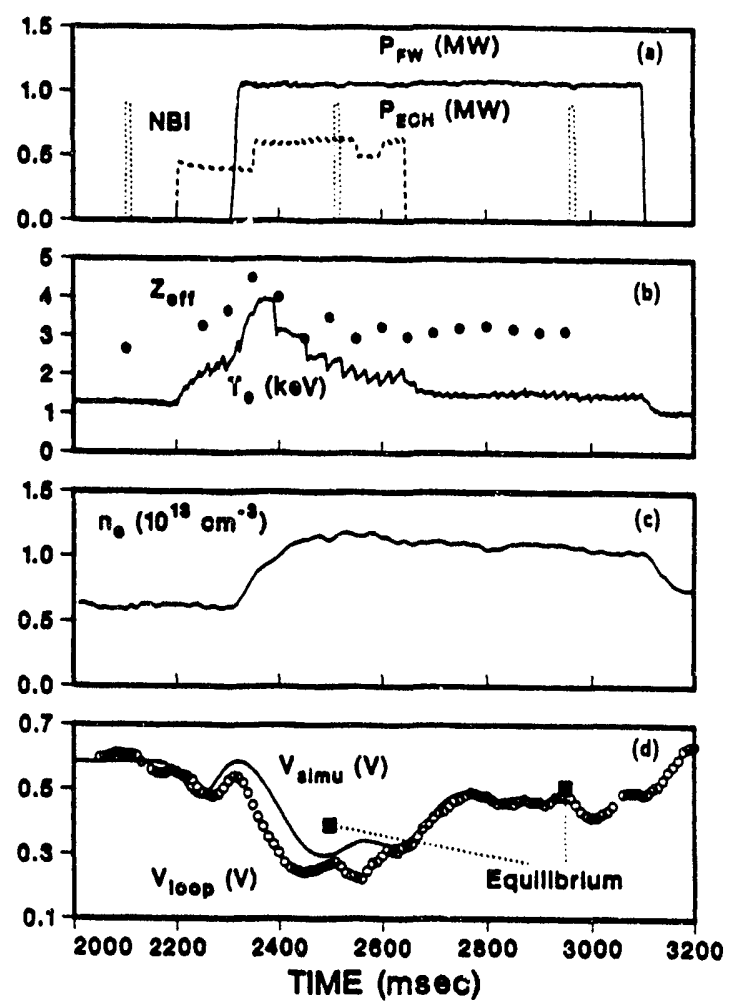

Fig. 9. Discharge with FWCD in the counter-current direction. The toroidal field is $1.1 \mathrm{~T}$ and the total plasma current is $397 \mathrm{kA}$. (a) Coupled FW and ECH power, and timing of injection of a $2 \mathrm{MW}$ neutral beam; (b) electron temperature by electron cyclotron emission from a normalized radius of 0.1 (solid line) and $Z_{\text {eff }}$ (circles); (c) line-averaged density; and (d) measured loop voltage corrected according to Eq. (8) (filled circles), surface voltage expected from a time-dependent simulation which does not include if current drive (solid line), and the loop voltage calculated from the profiles (assumed static) of $T_{e}, n_{e}$, and $Z_{e f f}$ (filled squares). 
Table I shows that during the ECH phase this process yields FWCD of $18 \pm 35 \mathrm{kA}$, compared with $-54 \mathrm{kA}$ expected from ray tracing calculations with the CURRAY code. For the FW case without ECH, the FWCD is $0 \pm 25 \mathrm{kA}$, compared to $-38 \mathrm{kA}$ from ray tracing.

It remains possible that the discrepancies l etween the measured current drive and the simulation could be reduced by improving the accuracy of the simulation in two key areas. First, ONETWO can use only a single magnetic equilibrium at present. Since the equilibrium changes when heating and current drive are introduced (since the pressure and current density profiles change), the ability to let the equilibrium evolve self-consistently is likely to be important. Second, the time-dependent effects of current drive need to be added to the simulation. The introduction of driven currents will induce local electric fields which tend to conserve the magnetic flux. These back-emfs will affect local Ohmic heating and modify the equilibrium and its behavior in time. Development of the capability to include these effects is underway.

\section{Discussion of Co- and Counter-FWCD}

Significant differences are found between co- and counter-FWCD which can be interpreted as the effect of adding or subtracting current density by FWCD near the center of the discharge. Figure 10 shows a comparison of the crash phase of two sawteeth, one from co-FWCD and one from counter-FWCD (the sawteeth near $2460 \mathrm{msec}$ in Figs. 4 and 9). This figure shows the change in the soft $x$-ray (SXR) intensity from just before to just aftar the crash for each of the diodes of the two SXR cameras, mapped onto the flux surface courdinate $\rho$. For the co-FWCD case, the sawtooth is normal: a large decrease in intensity takes place near the center and a large increase takes place outside the sawtooth inversion layer. The usual model is that the sawtonth causes magnetic reconnection resulting in a rapid flux of energy from the center across the inversion layer near the $q=1$ surface. In the counter-FWCD case the SXR channels behave the same outside the sawtooth inversion layer, but inside it the intensity pattern is quite different. Channels near the center actually increase during a sawtooth crash. The intensity pattern suggests that a second $q=1$ surface has been established inside the normal $q=1$ surface. This is consistent with the presence of a counter-current driven by the FW power, and earlier in time the partial suppression of sewteeth in the counter-FWCD case may be due to the driving of $q$ above unity at the plasma center by the negative currents driven near the center of the plasma.

The behavior of the internal inductance for co- and counter-FWCD also differs. As notel earlier, $\ell_{i}$ increases during co-FWCD, but it stays flat or slightly decreases during counter-FWCD. The behavior of both the sawteeth and the internal inductance are consistent with co-FWCD providing significant levels of central co-current drive and counter-FWCD providing small counter-current drive. The counter-FWCD discharges have higher density and lower electron temperature than the co-FWCD discharges, so the expected FWCD is smaller in the counter-FWCD cases. Figure 8 shows the calculated current density from FWCD for the co-FWCD and counter-FWCD cases of Figs. 4 and 9, respectively. In both cases the calculated current from FWCD lies almost completely within the $q=1$ surfaces.

An $x$-ray spectrometer which views the plasma perpendicularly through the center was used to study the electron distribution function. This spectrometer is sensitive to photons in the energy range of 10 to $60 \mathrm{keV}$. FW heating by itself at $1.1 \mathrm{~T}$ did not produce a tail for either direction of the fast waves, and the $x$-ray

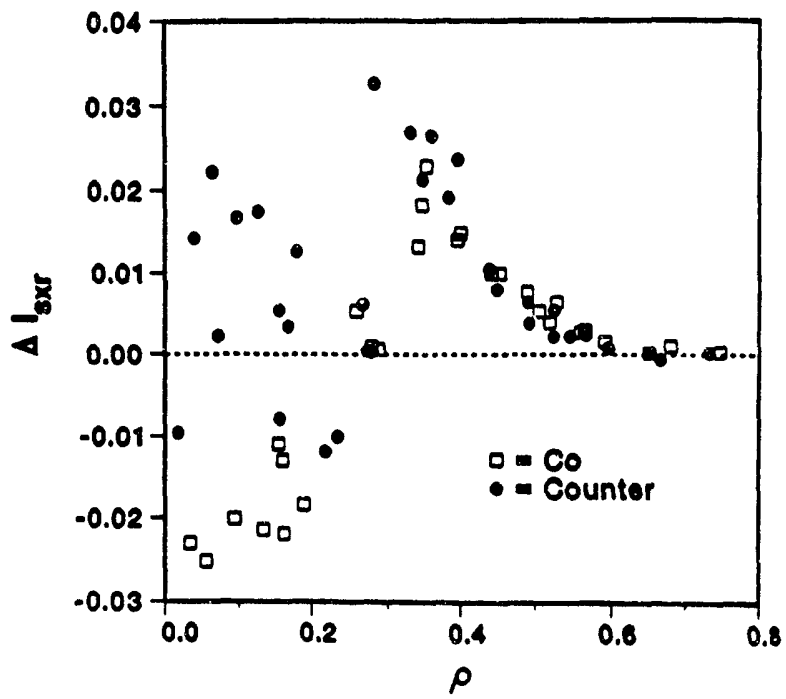

Fig. 10. Change in the emitted SXR intensity during a single sawtooth crash for two discharges, one with co-FWCD (empty squares) and one with counter-FWCD (filled circles). Each data point represents a single SXR detector mapped onto the flux function $\rho$. The toroidal field is $1.1 \mathrm{~T}$ and the total plasma current is $400 \mathrm{kA}$. 
temperature of the thermal spectrum was in agreement with other measures of electron temperature. With the application of $\mathrm{ECH}$, a strong tail with temperature of about $15 \mathrm{keV}$ is added to the distribution function, as shown in Fig. 11. For the case of co-FWCD, the addition of FWCD power enhances the magnitude of the tail without changing its temperature, while for counter-FWCD the FWCD power has almost no effect on the tail.

Fokker-Planck calculations using the CQL3D code (HARvEY, 1987) were performed to simulate the effect of combining FWCD with the effects of the Ohmic electric field. These calculations showed that a tail is developed on the distribution function by the OH field, and that enhancement of the tail by the FWCD is sensitive to the direction of the FWCD. However, the enhancement is significant only at relativistic energies where very few electrons are present. For example, at $500 \mathrm{keV}$ the distribution function is 10 times larger for co-FWCD than for counter-FWCD; however, the number of electrons at that energy is so small there is no effect on the conductivity of the plasma and no synergy between the OH field and the FWCD with respect to current drive is expected (HARVEY, 1992). On the basis of these results, it is concluded that the lack of symmetry between the co-FWCD and the counter-FWCD cannot be explained by distortions of the distribution function caused by the remaining dc electric field. This is so because the fast wave needs a high density of resonant electrons to overcome inherently weak damping. The Fokker-Planck code results agreed with the observation that without ECH no significant tail should be visible in the energy band to which the SXR analyzer is sensitive for either direction of FWCD.

\section{Summary of FWCD}

Up to about 100kA of plasma current has been driven by $1.1 \mathrm{MW}$ of FWCD power. ECH/ECCD helps to increase the FWCD, probably by increasing the electron temperature. The current drive is proportional
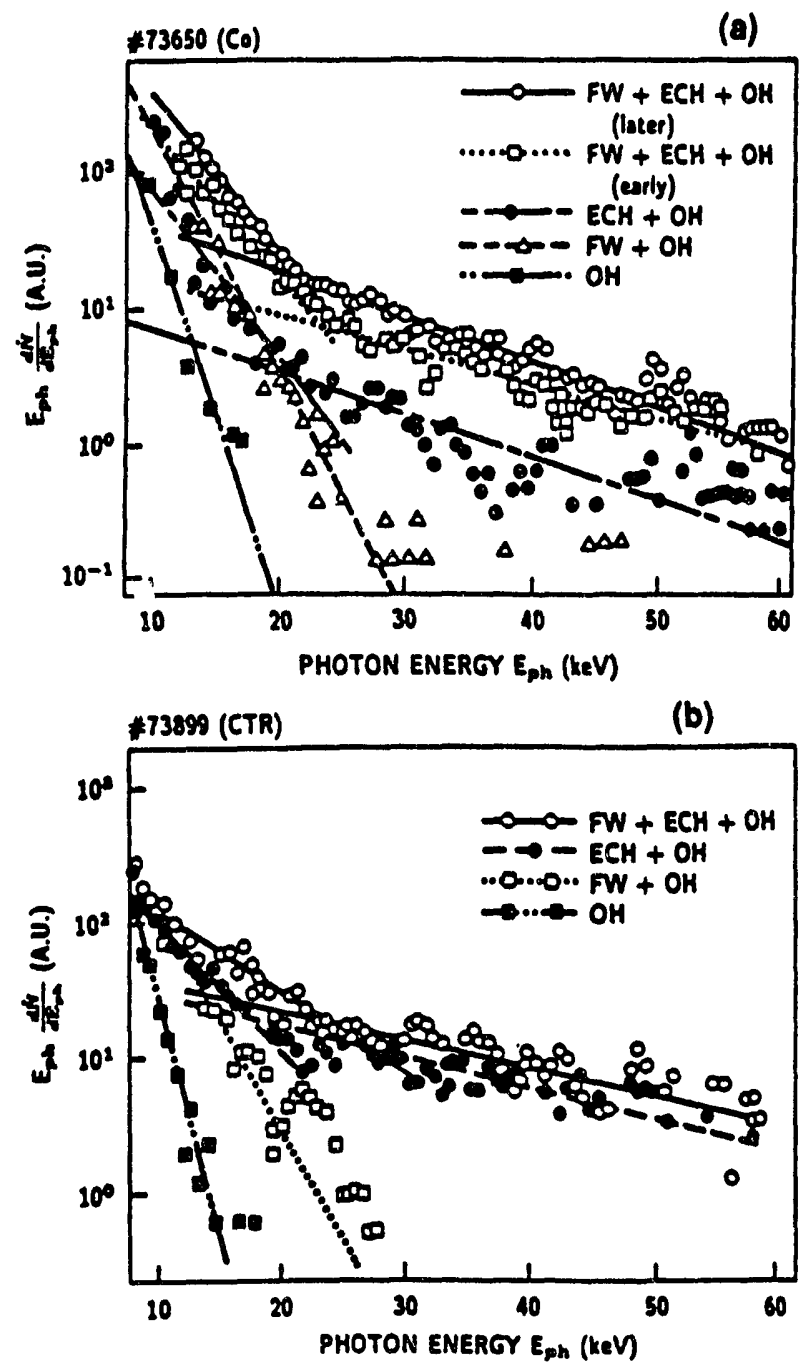

Fig. 11. Spectra of x-ray emission for the discharges of (a) Fig. 4 (co-FWCD) and (b) Fig. 9 (counter-FWCD). 
to the power per particle, and its magnitude agrees with a multiple-pass ray tracing calculation. Other details of the discharge which are sensitive to the profile of the current density, such as the behavior of the sawteeth and the internal inductance, seem qualitatively consistent with the calculated current drive.

For counter-FWCD discharges at $1 \mathrm{~T}$, the immediate effect of the FWCD is to suppress the sawteeth. This causes the electron temperature to rise uniii a large sawtooth occurs, at which time the electron temperature experiences a large sudden decrease. These changes in the electron temperature cause the Ohmic electric field at the surface of the plasma to be out of equilibrium with the field near the center of the plasma, and this difference must be compensated in order to evaluate the current drive. Doing so by means of a time-dependent simulation with a transport code yields an experimental determination of the driven current by counter-FWCD of $18 \pm 35 \mathrm{kA}$, compared to about $-50 \mathrm{kA}$ predicted by theory. The observed behavior of the sawteeth and the internal inductance is plausible for the expected FWCD.

The current drive efficiency for the co-FWCD case in these experiments is $\eta=0.014 \times 10^{20} \mathrm{MA} / \mathrm{MW} / \mathrm{m}^{2}$. It is anticipated that experiments at higher power will show that the efficiency increases strongly with electron temperature.

\section{ELECTRON CYCLOTRON CURRENT DRIVE}

Tise mechanism of electron cyclotron current drive (Fisch, 1980) is quite different from that of fast wave current drive. Electron cyclotron waves are absorbed by electrons through cyclotron damping, and they accelerate electrons in the direction perpendicular to the magnetic field. By virtue of their increased total energy, these electrons become less collisional. If the electron cyclotron wave with non-zero $k_{\|}$is dominantly absorbed on one side of the cyclotron resonance, electrons with parallel velocity in one toroidal direction will be preferentially heated (in the perpendicular direction). These electrons will experience less parallel friction with the ions due to their lower collinionality, so a net toroidal current will be generated as the distribution function relaxes. The current drive is substantially modified by the effect of trapping electrons in the magnetic well in toroidal geometry (OHKAWA, 1976).

The ECCD experiments on DIII-D satisfy the basic requirements of the theory: the damping of the electron ryclotron waves is strong enough that full absorption takes place on the antenna side of the cyclotron resonance, and the heated electrons are confined for much longer than their slowing down time. Reduced efficiency of ECCD in other experiments has been attributed to violation of these conditions.

Experiments on DIII-D have found currents of up to $100 \mathrm{kA}$ driven by ECCD. The experiments are performed using the techniques described in Section 4 for FWCD. Equation (5) is applied to ECH discharges with total current of 300 to $500 \mathrm{kA}$, densities of 1 to $2 \times 10^{10} \mathrm{~m}^{-3}$, electron temperature of 2.0 to $3.6 \mathrm{keV}$, $Z_{\text {eff }}$ of 2.5 to 5.5 , and toroidal field of $2 \mathrm{~T}$. These discharges are characterized by thermal distribution functions for electrons. In all cases, the current driven by ECCD is less than the total plasma current.

The current driven by ECCD is in the expected direction. Unlike the counter-FWCD case, the counterECCD appears to drive currents opposite the Ohmic current, although smaller in magnitude. In these experiments the If is launched in the same direction as the plasma current for co-ECCD, and counterECCD is accomplished by reversing the direction of the plasma current while keeping the ECH antennas in the same toroidal direction.

The Ohmic electric field is adjusted to regulate the current. This field is not zero because not all of the current is driven by ECCD. The residual de electric field can strongly affect the tail of the distribution function, especially under these low density conditions. Unlike the fast wave, the electron cyclotron wave can interact effectively with a low density tail. And inversely, the ECH can generate a perpendicular tail which can pitch angle scatter into the parallel direction and increase the conductivity of the plasma even for fixed temperature of the bulk of the distribution.

Accurate modeling of these discharges requires application of a Fokker-Planck code to evaluate the effects of the nonMaxwellian electron distribution. Studies with such a code show that the electric field is responsible for an increase in the apparent efficiency of ECCD of approximately $50 \%$ under the conditions of these experiments (JAMES, 1992). The important effect on the driven current of trapping of electrons in the magaetic well is also included in the Fokker-Planck calculations.

Good agreement is obtained between the experiment and the Folker-Planck theory. In Fig. 12 the experimental measurements of the ECCD are compared to the predictions of the theory. Good agreement, as shown, is obtained only when the effects of the dc electric field and the trapping of electrons is included. Other details of the discharges, such as the behavior of the electron cyclotron emission at high harmonics and the hard $x$-ray emission, tend to confirm the calculations of electron distribution.

For these discharges, the maximum efficiency of current drive is $\eta=0.016 \times 10^{20} \mathrm{MA} / \mathrm{MW} / \mathrm{m}^{2}$. This efficiency includes the effect of the remanent dc electric field, which increases the driven current by a factor of 2. 


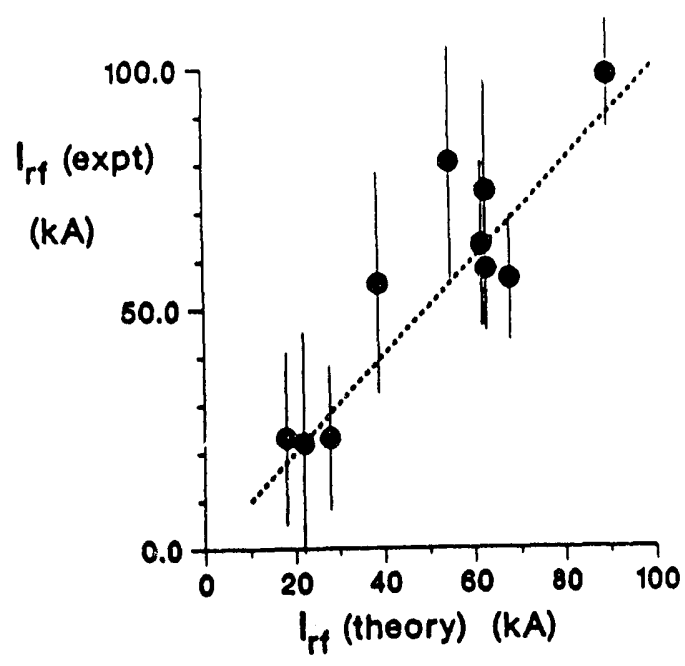

Fig. 12. Experimental messurements of the current driven by ECCD as a function of the ECCD current found from Fokker-Planck calculations including the effects of trapping and residual de electric field.

\section{NEUTRAL BEAM CURRENT DRIVE}

The first studies of neutral beam current drive in DIII-D were performed with hydrogen neutral beams (SIMONEN, 1988). The experiments were done by injecting $10 \mathrm{MW}$ of neutral beam power into a target plasms with $220 \mathrm{kA}$ of plasms current, density of $2 \times 10^{10} \mathrm{~m}^{-3}$, and toroidal field of $2.1 \mathrm{~T}$. At the start of the injection the applied Ohmic heating voltage was rapidly reduced to zero by holding constant the current in the $O H$ coil. The effect of the injection was to ramp the plasma current up to $340 \mathrm{kA}$ and maintain it for the duration of the heating pulse, 1.5 sec.

Estimates from computational modeling indicate that over $80 \%$ of the toroidal current was due to fast ion drive and that the fraction due to bootstrap current was between $10 \%$ and $20 \%$ of the total. The electron temperature was between 1 and $2 \mathrm{keV}$. The confinement time of $24 \mathrm{msec}$, normalized by the plasma current, is consistent with $\mathrm{H}$-mode scaling in DIII-D using hydrogen injection into deuterium plasmas with the same power level of neutral injection.

Recent experiments in DIII-D have utilized deuterium neutral beam injection into deuterium plasmas. These bave been performed to assess the influence of the improved confinement and higher beam power available with deuterium operation. In addition, the vacuum vessel walls have been coated with several hundred nanometers of boron compounds (JACKSON, 1992) which has acted to reduce the oxygen content in the plasma, reduce radiation from the plasma, and reduce $Z_{\text {eff }}$. These improvements have lead to higher electron temperatures and lower $Z_{\text {eft }}$, but the calculated neutral beam current drive is approximately the same as that obtained in the hydrogen experiments because the electron density was 2 to 3 times larger in the deuterium cace.

In the deuterium experiments, the Ohmic heating coil is open circuited at a point in time when the current in the coil is near zero. Figure 13 shows that as the current in the Ohmic coil is ramped toward zero, the plasms current is reduced from 1.8 to $1.3 \mathrm{MA}$. This reduction removes current from the outer regions of the plasma, increasing $l_{i}$ from 0.85 to 1.2 .

Starting at $1500 \mathrm{msec}$ the plasma current decays inductively with only minimal current drive (less than $40 \mathrm{kA}$ ) from the single injected neutral beam. At $2500 \mathrm{msec}$ when full beam power is applied, the plasma current increases by about $100 \mathrm{kA}$ for the first $150 \mathrm{msec}$ and then begins to decay at about $30 \mathrm{kA} / \mathrm{sec}$ for the remainder of the high power phase. The negative surface voltage present during the current ramp when the beams come on reduces the magnitude of the rise in current due to NBCD. The negative surface voltage is due mainly to the increase in major radius which accompanies heating. Since the plasma current increases by $100 \mathrm{kA}$ even with a negative surface voltage, it can be concluded that the NBCD is greater than $100 \mathrm{kA}$.

Beginning at $3000 \mathrm{msec}$, the plasma conditions have developed into a quasi-stationary state. Using kinetic profile data at $3100 \mathrm{msec}$, a computational modeling of the plasma indicates $350 \mathrm{kA}$ of neutral beam current drive, $340 \mathrm{kA}$ of bootstrap current, and $500 \mathrm{kA}$ of residual inductive current remaining from the fully inductive phase of the discharge. The calculations of NBCD and bootstrap current are in agreement with the formulisms developed during the conceptual design activity of ITER.

The decay in plamma current is consistent with the view that about $500 \mathrm{kA}$ of the total plasma current is inductively driven. With a calculated $L / R$ time of $30 \mathrm{sec}, 500 \mathrm{kA}$ of residual inductive current should result in a plasma current decay rate of about $17 \mathrm{kA} / \mathrm{sec}$ and a surface voltage of about $40 \mathrm{mV}$. Analysis of the 


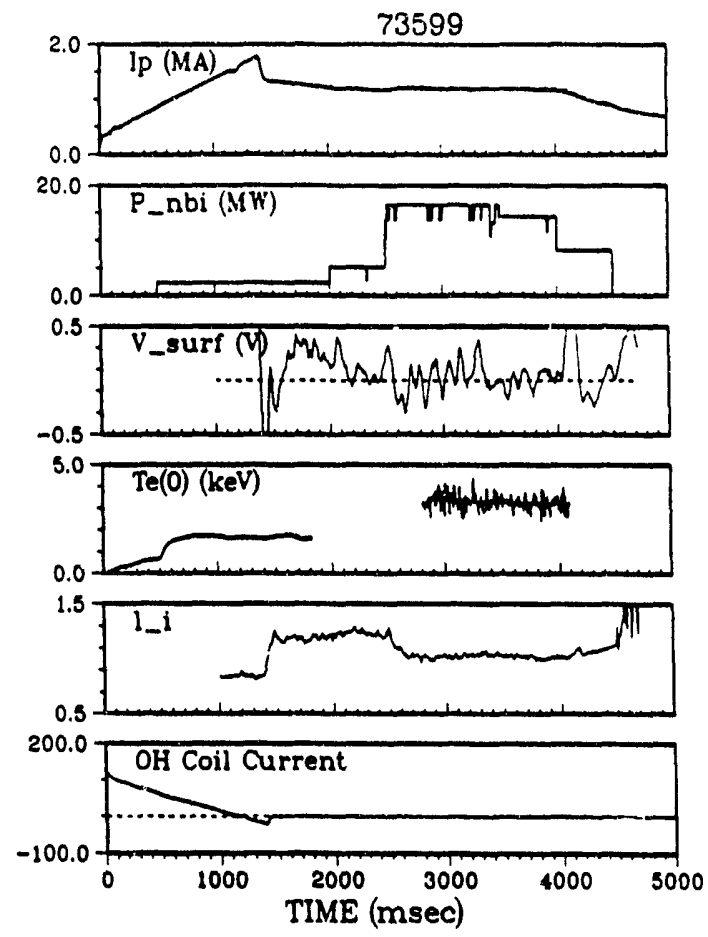

Fig. 13. Discharge with neutral beam current drive. The toroidal field is $2.1 \mathrm{~T}$, the working gas and the injection species is deuterium, and the density is $6 \times 10^{10} \mathrm{~m}^{-3}$ and $Z_{\text {eff }}$ is 2.25 during the high power phase. Part of the trace of electron temperature is missing due to cutoff of the electron cyclotron emission near the center.

currents flowing in the equilibrium coils shows that the currents are changing in such a manner as to induce a voltage of appraximately $-40 \mathrm{mV}$ at the surface of the plasma. This explains the measured net surface voltage of approximately zero volts for about $1.5 \mathrm{sec}$ during the high power beam phase. In addition, the voltage induced by the equilibrium coils is probably responsible for the faster than expected decay of the plasma current.

In conclusion, we can state that although the results of these experiments are far from definitive, the modeling and analysis suggests that the calculated current drive of $350 \mathrm{kA}$ and the measurements of $>100 \mathrm{kA}$ to $<500 \mathrm{kA}$ are not inconsistent.

\section{CONCLUSIONS}

This comparison of current drive in DIII-D using three different techniques for current drive shows that the techniques have remarkably similar efficiency. Table II shows a comparison of the cases presented here. For electron temperature in the range 2 to $3.5 \mathrm{keV}$, the current drive efficiency is $0.015 \pm 0.003 \mathrm{MA} / \mathrm{MW} / \mathrm{m}^{2}$ for FWCD, FWCD with ECH heating, ECCD, and NBCD.

This work needs to be extended to higher power for the rf techniques, to be conclusive. Higher power has the additional advantage that, given suitable wave launchers which match the wave velocity to the electron velocity, the current drive efficiency improves with electron temperature, which also rises with power.

TABIE II

Comparison of the Current Drive Methods Applied to DIII-D

\begin{tabular}{lccccc}
\hline & $\begin{array}{c}I_{p} \\
(\mathrm{MA})\end{array}$ & $\begin{array}{c}n_{e} \\
\left(10^{19} \mathrm{~m}^{-3}\right)\end{array}$ & $\begin{array}{c}T_{i} \\
(\mathrm{keV})\end{array}$ & $\begin{array}{c}P \\
(\mathrm{MW})\end{array}$ & $\begin{array}{c}\eta \\
\left(10^{20} \mathrm{MA} / \mathrm{MW} / \mathrm{m}^{2}\right)\end{array}$ \\
\hline FWCD & 0.4 & 1 & 2.0 & 1 & 0.014 \\
FWCD + ECH & 0.4 & 1 & 3.5 & 1.5 & 0.014 \\
ECCD & 0.4 & 1 & 3.0 & 1 & 0.016 \\
NBCD $(\mathrm{H} \rightarrow \mathrm{He})$ & 0.3 & 2 & 2.0 & 10 & 0.011 \\
NBCD $(\mathrm{D} \rightarrow \mathrm{D})$ & 1.2 & 6 & 3.3 & 16 & 0.018 \\
\hline
\end{tabular}




\section{ACKNOWLEDGEMENTS}

The authors would like to thank the DIII-D operations and physics teams for excellent support in the course of this work. This work was supported by the U.S. Department of Energy under Contract Nos. DE-AC03-89ER51114, DE-AC03-78ET51013, DE-AC02-76-CHO-3073, and DE-AC05-84OR21400.

\section{REFERENCES}

AKaokA N., et al. (1991) Plasma Physics and Cont. Nucl. Fusion Research, Washington, 1990, Vol. I, p. 53. International Atomic Energy Asssociation, Vienna.

Bonoli P.T., and Porkolab M. (1992) in American Institute of Physics Conference Proceedings 244 Radio Frequency Power in Plasmas, Charleston, South Carolina 1991 (edited by DoNALD B. BATCHELOR), p. 155. American Institute of Physics, New York.

Bonoli P.T., Porkolab M., Takase Y., Knowlton S.F., (1988) Nucl. Fugion $28,991$.

Carlstrom T.N. et al. (1989) in Proceedings of 16 th European Conference on Controlled Fusion and Plasma Physics, Venice (edited by S. Segre, H. KnOePfel, AND E. Sindoni), Vol. 13B, Part 1, p. 241. European Physical Society, Geneva.

Chiv, S.C., Chan V.S., Harvey R.W., Porkolab M. (1989) Nucl. Fusion $29,2175$.

ChIU S.C. et al. (1992) in Proceedings of Europhysics Topical Conf. on Fiadiofrequency Heating and Current Drive of Fusion Devices, Brussels 1992 (edited by C. Gormezano, P.U. LAMalle, R.R. Weynants), Vol. 16E, p. 177. European Physical Society, Geneva.

Fisch N.J., Boozer A.H. (1980) Phys. Rev. Letters 45, 720.

Fisch N. (1987) Rev. Mod. Physics 59, 175.

Gouldivg R.H. et al. (1992) in American Institute of Physics Conference Proceedings 244 Radio Frequency Power in Plasmes, Charleston, South Carolina 1991, (edited by DONALD B. BATCheloR), p. 287. American Institute of Physics, New York.

Groebner R.J., Peebles W.A. et al. (1991) in Plasma Physics and Cont. Nucl. Fusion Research, Washington, 1990, Vol. 1, p. 453. International Atomic Energy Association, Vienna.

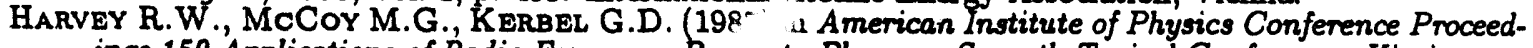
ings 159 Applications of Radio Frequency Power to Plasmas, Seventh Topical Conference, Kissimmee, Florida 1987, (edited by S. BerNaBer and R.W. MOTLEY), p. 49. American Institute of Physics, New York.

Harvey R.W., et al. (1992) in Proceedings of the International Atomic Energy Association Technical Committee Meeting on Fast Wave Current Drive in Reactor Scale Tokamaks, Arles, France, September 29-25, 1991, p. 135. EURATOM-CEA, St Paul lez Durance.

HoFFMAN D.J. et al. (1992) in Proceedings of the International Atomic Energy Association Technical Committee Meeting on Fast Wave Current Drive in Reactor Scale Tokamaks, Arles, France, September 23-25, 1991, p. 456. EURATOM-CEA, St Paul lez Durance.

ITER TEAM (1989) "ITER Conceptual Design-Interim Report." International Atomic Energy Association, Vienna.

ITER TEAM (1991) "ITER Conceptual Design Report," IAEA/ITER/DS/18. International Atomic Energy Association, Vienna.

JACKson G.L., WINTER J. et al. (1991) Phys. Rev. Letters 67, 3098.

JACKSON G.L. et al. (1992) "Very High Confinement Discharges in DIII-D after Boronization," to be published in Phys. Fluids $B$.

JAMES R. et al. (1990) in Europhysics Conference Abstracts (edited by G. BRIFFOD and F.C. SCHÜLLER), Vol. 14B, Part III, p. 1259. European Physical Society, Amsterdam.

JAMEs R.A. et al. (1992) Phys. Rev. A 45, 8783.

Lao L.L., St.John H., Stambaugh R.D., Kellman A.G., and Pfeiffer W. (1985) Nucl. Fusion 25, 1611.

LUCE T.C. et al. (1991) in Plasma Physics and Cont. Nucl. Fusion Research, Washington, 1990, V. 1, p. 631. International Atomic Energy Association, Vienna.

MayberRy M.J., Pinsker R.I., PETTY C.C. et al. (1992) in American Institute of Physics Conference Proceedings 244 Radio Frequency Power in Plasmas, Charleston, South Carolina, 1991 (edited by Donald B. Batchelor), p. 276. American Institute of Physics, New York.

Meade D.M. et al. (1991) in Plasma Physics and Cont. Nucl. Fusion Research, Washington, 1990, Vol. 1, p. 9. International Atomic Energy Association, Vienna.

Navratil G.A. et al. (1990) in Plasma Physics and Cont. Nucl. Fusion Research, Washington, 1990, Vol. 1, p. 209. International Atomic Energy Association, Vienna.

OHKAWA T. (1976) General Atomics Report GA-A13847.

PETTY C.C. et al. (1992a) in American Institute of Physics Conference Proceedings 241 Radio Frequency Power in Plasmas, Charleston, South Carolina, 1991 (edited by DONALD B. BATCheloR), p. 96. American Institute of Physics, New York.

Petry C.C. et al. (1992b) Phys. Rev. Lett. 69, 289.

PETTY C.C. et al. (1992c) in Proceedings of Europhysics Topical Conference on Radiofrequency Heating and Current Drive of Fusion Devices, Brussels, 1992 (edited by C. Gormezano, P.U. LAMALLE, R.R. Weynants), Vol. 16E, p. 177. European Physical Society, Geneva. 
PETTY C.C. et al. (1992d) in American Institute of Physics Confererwe Proceedings 244 Radio Frequency Power in Plasmas, Charleston, South Carolina 1991, (edited by Donald B. BATCheloR), p. 133. American Institute of Physics, New York.

PINSKER R.I. et al. (1992) in Proceedings of the 14th IEEE Symp. on Fusion Engineering, San Diego, 1991, p. 115. IEEE, Piscataway, New Jersey.

Porkolab M. (1992) in American Institute of Physics Conference Proceedings 244 Radio Frequency Power in Plasmas, Charleston, South Carolina 1991 (edited by Donald B. Batchelor), p. 197. American Institute of Physics, New York.

Prater R. et al. (1992) in Proceedings of the International Atomic Energy Association Technical Committee Meeting on Fast Wave Current Drive in Reactor Scale Tokamaks, Arles, France, September 23-25, 1991, p. 308. EURATOM-CEA, St Paul lez Durance.

Robinson D.C. et al. (1987) in Plasma Physics and Cont. Nucl. Fusion Research, Kyoto, 1986, paper F-III-2. International Atomic Energy Association, Vienna.

Simonen T.C., Matsuoka M. et al. (1988) Phys. Rev. Letters 61, 1720.

Söldner F.X. et al. (1991) in Plasma Physics and Cont. Nucl. Fusion Research, Washington, 1990, Vol. 1, p. 613. International Atomic Energy Associaton, Vienna.

START D.F.H. et al. (1992) in Proceedings of the International Atomic Energy Association Technical Con:mittee Meeting on Fast Wave Current Drive in Reactor Scale Tokamaks, Arles, France, September 23-25, 1991, p. 227. EURATOM-CEA, St Paul lez Durance.

Start D.F.H. et al. (1982) Phys. Rev. Letters 48, 624.

StraIT E.J. et al. (1991) 18th European Conference on Controlled Fusion and Plasma Physics, Berlin, 3-7 June 1991, Vol. II, p. 105. European Physical Society, Geneva.

TANAKa H. et al. (1988) Phys. Rev. Letters 60, 1033.

Taylor T.S., Lazanus, E.A. et al. (1991) in Plasma Physics and Cont. Nucl. Fusion Research, Washington, 1990, Vol. 1, p. 177. International Atomic Energy Association, Vienna.

Uehara K., Krimura H. et al. (1989) in Radio Frequency Power in Plasmas, Eighth Topical Conference, Irvine, Californio 1989, (edited by Roger MCW ILliaMs) p. 106. American Institute of Physics, New York.

Van Nieunienhove R., Koch R., Van Oost G. (1990) Nucl. Fusion 31, 1770.

VAN Nieuwenhove R. et al. (1992) "Comparison of the Performance of an ICRF Antenna with and without Faraday Shield on Textor," submitted for publication in Nucl. Fusion.

Wagner F. et al. (1982) Phys. Rev. Letters 49, 1408.

Zarnstorf M.C. et al. (1988) Phys. Rev. Letters 60, 1306. 

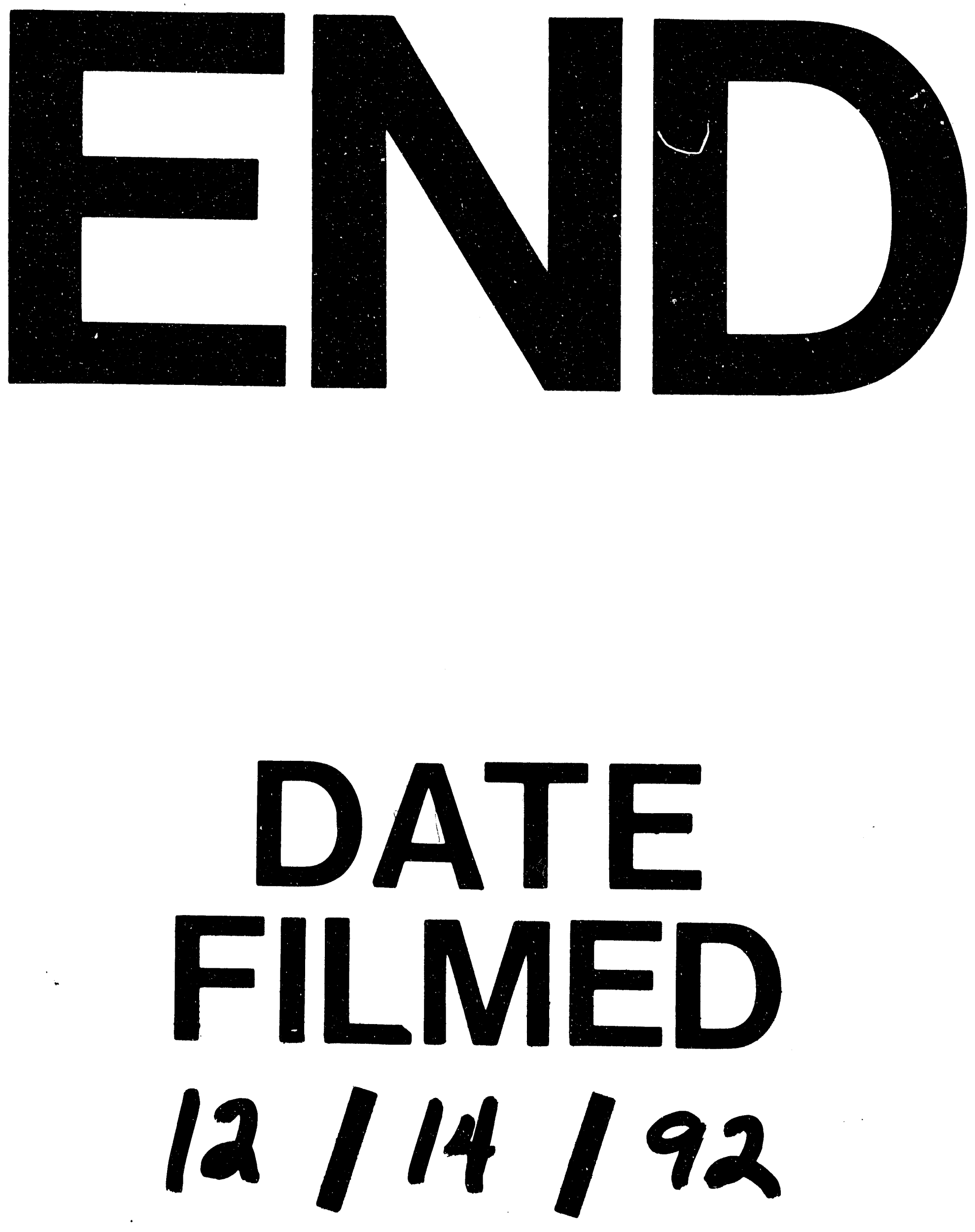
